\title{
Implementing a Particle-Fluid Model of Auroral Electrons
}

\author{
Jörgen Vedin and Kjell Rönnmark \\ Department of Physics, Umeå University, SE-901 87 Umeå, Sweden \\ \{jorgen.vedin, kjell.ronnmark\}@space.umu.se
}

\begin{abstract}
The particle-fluid model of auroral electrons that is presented in [1] is a major step forward within the field of dynamic models of the auroral generation mechanisms. The model is, however, also an example where the implementation of a physical model requires a lot of knowledge from the field of computer science. Therefore, this paper contains a detailed description of the implementation behind the particlefluid model. We present how the particles are implemented in doubly linked lists, how the fluid equations are solved in a time-efficient algorithm, and how these two parts are coupled into a single framework. We also describe how the code is parallelized with an efficiency of nearly $100 \%$.
\end{abstract}

\section{Introduction}

The aurora is created by electrons that are accelerated along the Earth's magnetic field lines before they impinge on the ionosphere and create light through excitation processes. To model the large-scale processes involved in the generation of auroras it is common to describe the electrons as a charged fluid, where the fluid equations are solved self-consistently together with Maxwell's equations. However, as the electrons are accelerated they are also heated in a process that is not properly described by a fluid model. Therefore, we have in [1] introduced a particle-fluid model of the auroral electrons, where the field solver is complemented by a particle pusher. By letting the electric field from the field solver accelerate the particles, and then using the temperature of the particle distribution as a feed-back to the field solver, we obtain a self-consistent description of the auroral electrons.

The physical description of the model is given in [1], and in this paper we concentrate on the details of the implementation. We will describe how the set of equations in the field solver are solved using an implicit algorithm in which the discretized equations are rewritten on a block-tridiagonal form to achieve good performance. We will also discuss how the particle data is implemented in linked lists to obtain a time-efficient algorithm, and how the particles and the field solver are coupled in a parallelized code. First, however, we will in the next section describe the basics of the model. 


\section{Model}

We use a two-dimensional model where the coordinates are $z$ along the Earth's magnetic field and $x$ spanning different latitudes, as can be seen in Fig. 1. The dynamics of auroral electrons is in real life controlled by a generator mechanism located in the tail of the Earth's magnetosphere. To mimic this process we prescribe a generator force in our model. The generator creates an ion current perpendicular to the magnetic field lines. At the flanks of the generator region the perpendicular current is diverted into field-aligned currents connecting the generator to the ionosphere. At the ionosphere these field-aligned currents are closed by a perpendicular ion current. Thus, we have a current circuit where the upward field-aligned current is carried by downgoing electrons that create auroras.

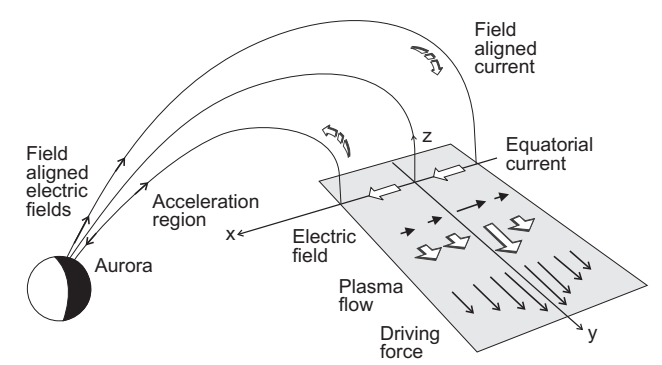

Fig. 1. The geometry of the auroral current circuit and the generator region in the equatorial magnetosphere. The curvature of the magnetic field lines is neglected in our model equations, but the convergence of the magnetic field lines is retained.

\section{$2.1 \quad$ Field Solver}

The electron fluid is in our model described by the equations

$$
\begin{gathered}
\partial_{\mathrm{t}} \mathrm{E}_{\mathrm{x}}=-\mathrm{A}^{2} \partial_{\mathrm{z}} \mathrm{B}_{\mathrm{y}}-\left(1-\mathrm{A}^{2}\right) \mathrm{F} \\
\partial_{\mathrm{t}} \mathrm{E}_{\mathrm{z}}=\frac{B_{z}}{B_{0}}\left(\partial_{\mathrm{x}} \mathrm{B}_{\mathrm{y}}+\mathrm{j}_{\mathrm{z}}\right) \\
\partial_{\mathrm{t}} \mathrm{B}_{\mathrm{y}}=\partial_{\mathrm{x}} \mathrm{E}_{\mathrm{z}}-\partial_{\mathrm{z}} \mathrm{E}_{\mathrm{x}} \\
\partial_{\mathrm{t}} \mathrm{n}=-\partial_{\mathrm{z}} \mathrm{j}_{\mathrm{z}} \\
\partial_{\mathrm{t}} \mathrm{j}_{\mathrm{z}}=-\frac{m_{i}}{m} \mathrm{nE}_{\mathrm{z}}-\partial_{\mathrm{z}}\left(\mathrm{nT}_{\mathrm{z}}+\frac{\mathrm{j}_{\mathrm{z}}^{2}}{\mathrm{n}}\right)-\mathrm{nT} \mathrm{T}_{\perp} \frac{\partial_{\mathrm{z}} B_{z}}{B_{z}}
\end{gathered}
$$

which are the non-zero components of Maxwell's equations together with the equation of continuity and the momentum equation. Here we use simulation variables, where $E_{x}$ and $E_{z}$ are the electric fields, $B_{y}$ is the perpendicular magnetic 
field, $\mathrm{A}$ is the Alfvén velocity, $\mathrm{F}$ is the generator force, $\mathrm{n}$ is the electron density, $j_{z}$ is the field-aligned electron current, while $T_{z}$ and $T_{\perp}$ are the field-aligned and perpendicular temperatures. $B_{z}(z)$ is the geomagnetic field and $B_{0}$ is the field strength at $z=0$ in the equatorial plane. The mass ratio in the last equation is the ion mass $m_{i}$ divided by the electron mass $m$. For an extensive derivation of these equations and a detailed description of the physics they describe, the reader is referred to [1]. Notice that the ion dynamics is neglected in the present version of the model. This introduces constraints on the generator, and we choose its length and time scales to be larger than the ion gyroradius and the ion gyroperiod respectively.

The equation system in (1) is underdetermined since the time evolution of the temperatures $T_{z}$ and $T_{\perp}$ is not included. To close the equation system, we therefore introduce a particle pusher from which we can obtain temperatures that are consistent with the field solver in each time step.

\section{$2.2 \quad$ Particle Pusher}

The field solver that solves equations (1) is coupled to a particle pusher according to the cartoon in Fig. 2. This cycle is performed for each time step in the simulation. The particles are accelerated by the electric field from the field

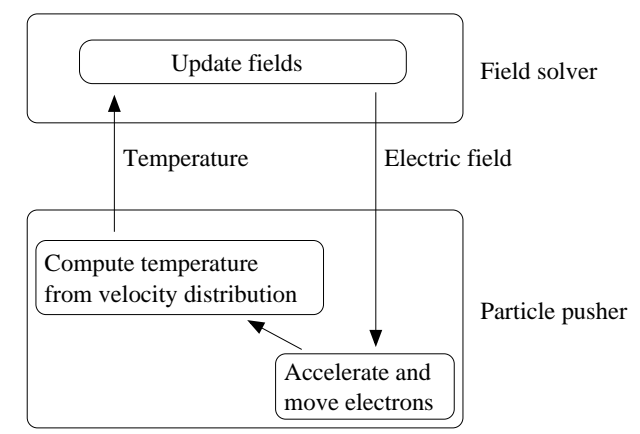

Fig. 2. Cycle performed for each time step to couple the field solver and the particle pusher.

solver, which implies that the particles move consistently with the fluid's evolution. From the velocity distribution of the electrons we can then determine temperatures that can be used in the momentum equation (1e).

The particles move along $z$ with velocity $v_{z}$ and gyrate the field line with velocity $v_{\perp}$. Their position and velocity are updated according to

$$
\begin{aligned}
d_{t} z & =v_{z}, \\
d_{t} v_{z} & =\frac{-e}{m} E_{p}-\mu \partial_{z} B_{z},
\end{aligned}
$$


where $-e$ is the electron charge, $\mu=m v_{\perp}^{2} / 2 B_{z}$ is the conserved magnetic moment of the particle, and $E_{p}$ is the electric field that accelerates the particle. The electric field $E_{p}$ is equal to the field-aligned electric field in the field solver, but with a correction described in [1] to ensure that the density and current of the particles are equal to the density and current of the fluid.

\section{Implementation}

\subsection{Grid}

The model is implemented on a two-dimensional grid of mesh size $n_{x} \times n_{z}$ illustrated in Fig. 3, where the generator boundary is at $z=0$ and the ionosphere is on the opposite side of the simulation region. Since the $z$-coordinate is aligned with the magnetic field and the magnetic field lines are converging as they approach the ionosphere, the grid point separation $\Delta x$ must decrease towards the ionosphere. The size of the simulation region is $L_{z}=55,000 \mathrm{~km}$ in the $z$-direction and $L_{x}=4,600 \mathrm{~km}$ in the $x$-direction at the generator boundary. The system length in the $x$-direction at the ionospheric boundary is merely $200 \mathrm{~km}$. The grid is also chosen to be inhomogeneous for resolving the interesting features of auroral acceleration that take place mainly at altitudes below $10,000 \mathrm{~km}$ $(z>45,000 \mathrm{~km})$ and at the field lines close to $x=0$. The typical mesh size

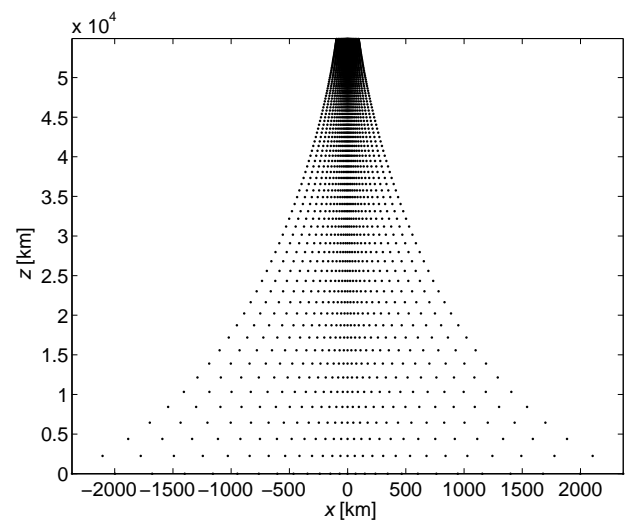

Fig. 3. The inhomogeneous grid on which the model is implemented with the generator boundary at $z=0$ and the ionosphere on the opposite side of the simulation region.

in our simulations is $n_{x} \times n_{z}=27 \times 100$. The value of $n_{x}$ is chosen to resolve current filaments with a width of a few kilometers at the ionospheric boundary, while the value of $n_{z}$ is large enough to get a good resolution of the acceleration region which has a length of a few thousand kilometers along $z$ at an altitude centered about roughly $6,000 \mathrm{~km}$. 


\section{$3.2 \quad$ Field Solver}

The set of equations in (1) is solved by a time-centered implicit method, based on factorization of the two-dimensional spatial differential operators. Algorithms of this type are discussed by, for example, [3]. If we for notational convenience collect all the fields in a vector $\mathbf{U}=\left(\mathrm{E}_{\mathrm{x}}, \mathrm{E}_{\mathrm{z}}, \mathrm{B}_{\mathrm{y}}, \mathrm{n}, \mathrm{j}_{\mathrm{z}}\right)^{T}$, we can after time discretization sum up (1) in the form

$$
\mathbf{U}(t+\Delta t)=\mathbf{U}(t)+\Delta t\{\mathbf{Q}(\mathbf{U}(t+\Delta t / 2))+\mathbf{G}\},
$$

where $\mathbf{Q}(\mathbf{U})$ represents the $\mathbf{U}$ dependence of the right hand side of (1) and $\mathbf{G}$ represents the inhomogeneous term involving the generator force $\mathrm{F}$. We now linearize $\mathbf{Q}$ in $\mathbf{U}(t+\Delta t)$ by a Taylor expansion:

$$
\begin{aligned}
& \mathbf{Q}(\mathbf{U}(t+\Delta t / 2)) \\
\approx & \mathbf{Q}(\mathbf{U}(t))+\frac{1}{2}[\mathbf{U}(t+\Delta t)-\mathbf{U}(t)] \cdot \partial_{\mathbf{U}} \mathbf{Q}(\mathbf{U}(t)) \\
= & \frac{1}{2}[\mathbf{U}(t+\Delta t)+\mathbf{U}(t)] \cdot \partial_{\mathbf{U}} \mathbf{Q}(\mathbf{U}(t)),
\end{aligned}
$$

where the second step follows from the homogeneous properties of $\mathbf{Q}$. This results in a linear set of equations:

$$
\left[\mathbf{I}-\frac{\Delta t}{2} \partial_{\mathbf{U}} \mathbf{Q}\right] \cdot \mathbf{U}(t+\Delta t)=\left[\mathbf{I}+\frac{\Delta t}{2} \partial_{\mathbf{U}} \mathbf{Q}\right] \cdot \mathbf{U}(t)+\Delta t \mathbf{G} .
$$

The operator $\partial_{\mathbf{U}} \mathbf{Q}$, which contains both $\partial_{x}$ and $\partial_{z}$, is now split into two parts as $\partial_{\mathbf{U}} \mathbf{Q}=\mathbf{X}+\mathbf{Z}$, where $\mathbf{X}$ contains only $\partial_{x}$ and $\mathbf{Z}$ contains only $\partial_{z}$. This decomposition is not unique, and it should be chosen in a way that makes the product $\mathbf{X} \cdot \mathbf{Z}$ as small and simple as possible. Neglecting the term $\Delta t^{2} / 4 \mathbf{X} \cdot \mathbf{Z}$. $[\mathbf{U}(t+\Delta t)-\mathbf{U}(t)]$, which is of third order in $\Delta t$, we can factorize the terms in equation (6) as in an alternating direction implicit (ADI) method to find

$$
\begin{aligned}
& {\left[\mathbf{I}-\frac{\Delta t}{2} \mathbf{X}\right] \cdot\left[\mathbf{I}-\frac{\Delta t}{2} \mathbf{Z}\right] \cdot \mathbf{U}(t+\Delta t)=} \\
& {\left[\mathbf{I}+\frac{\Delta t}{2} \mathbf{X}\right] \cdot\left[\mathbf{I}+\frac{\Delta t}{2} \mathbf{Z}\right] \cdot \mathbf{U}(t)+\Delta t \mathbf{G} .}
\end{aligned}
$$

Introducing $\mathbf{U}^{*}=[\mathbf{I}-\Delta t / 2 \mathbf{Z}] \cdot \mathbf{U}(t+\Delta t)$ as a new variable, we can solve (7) in two steps. First we solve

$$
\begin{aligned}
& {\left[\mathbf{I}-\frac{\Delta t}{2} \mathbf{X}\right] \cdot \mathbf{U}^{*}=} \\
& {\left[\mathbf{I}+\frac{\Delta t}{2} \mathbf{X}\right] \cdot\left[\mathbf{I}+\frac{\Delta t}{2} \mathbf{Z}\right] \cdot \mathbf{U}(t)+\Delta t \mathbf{G}}
\end{aligned}
$$

for $\mathbf{U}^{*}$. Then we use

$$
\left[\mathbf{I}-\frac{\Delta t}{2} \mathbf{Z}\right] \cdot \mathbf{U}(t+\Delta t)=\mathbf{U}^{*}
$$


to solve for the fields at $t+\Delta t$. When the operators $\mathbf{X}$ and $\mathbf{Z}$ are expressed as centered finite differences, each of these two steps consists of solving a blocktridiagonal set of equations. The number of operations needed for this scales linearly with the mesh size $\left(n_{x} \times n_{z}\right)$ and hardly requires any extra memory, which makes this algorithm very efficient. The number of operations needed for a direct integration of (6) would typically be proportional to $\left(n_{x} \times n_{z}\right)^{2}$.

\subsection{Particle Pusher}

In this model the particles are used roughly as in a regular Particle-In-Cell (PIC) code, see for example [2]. The input to the particle pusher is the electric field computed in the field solver, and the output from the particle pusher is the temperatures $T_{z}$ and $T_{\perp}$. The electric field and the temperatures are given on the grid points, but the particles can of course be located also in-between two grid points. To handle this, a PIC code utilizes a weight scheme, and in this model we use linear weights. The electric field (and also the magnetic field) used in (3) is determined from the fields at the two grid points that are closest to the particle's position, as can be seen in Fig. 4 . At the particle's position $z_{i}$, the

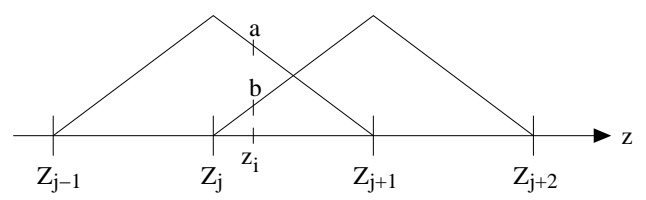

Fig. 4. To determine the electric field at the particle position $z_{i}$ we use triangular shape functions. The electric field at grid point $Z_{j}$ is weighted by the value of the shape function in point $a$ and the electric at grid point $Z_{j+1}$ is weighted by the value of the shape function in point $b$. These two values are then assigned to the electric field $E\left(z_{i}\right)$.

electric field is given by

$$
E\left(z_{i}\right)=\left(\frac{Z_{j+1}-z_{i}}{\Delta z}\right) E_{j}+\left(\frac{z_{i}-Z_{j}}{\Delta z}\right) E_{j+1},
$$

where $E_{j}$ and $E_{j+1}$ are the electric field values at the grid points $Z_{j}$ and $Z_{j+1}$, while $\Delta z$ is the grid point separation. When the temperatures are computed, the weighting is inverted, and the temperatures at a grid point get a contribution from all particles located in the two grid cells surrounding the grid point, as can be seen in Fig. 5. If $T_{i}$ is the contribution to the temperature from a particle located at $z_{i}$, then

$$
T_{j}=\left(\frac{Z_{j+1}-z_{i}}{\Delta z}\right) T_{i}
$$




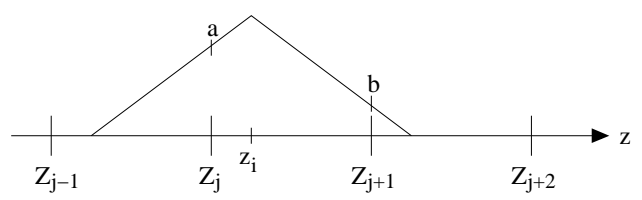

Fig. 5. A particle at position $z_{i}$ will contribute to the temperatures at grid points $Z_{j}$ and $Z_{j+1}$. If $T_{i}$ is the contribution to the temperature from a particle located at $z_{i}$, then $T_{i}$ will be weighted by the shape function's value at $a$ before it is added to the temperature at $Z_{j}$ and it will be weighted by the shape function's value at $b$ before it is added to the temperature at $Z_{j+1}$.

is the part of $T_{i}$ that is added to the temperature at grid point $Z_{j}$. Correspondingly the temperature at $Z_{j+1}$ gets the contribution

$$
T_{j+1}=\left(\frac{z_{i}-Z_{j}}{\Delta z}\right) T_{i}
$$

from a particle at $z_{i}$.

During the simulation, particles that move outside the system boundaries are removed and new particles are injected at a rate determined to maintain a constant density and temperature at the boundaries. To handle this adding and removing of particles in a sufficiently fast manner the particles are implemented as a linked list. Actually, we implement the particles as six linked lists. There are two types of particles, particles of magnetospheric origin that are injected at the generator boundary and particles of ionospheric origin that are injected at the ionospheric boundary. Each type of particles are stored in three separate lists. The particles that are active in the simulation are stored in an inside-list, while the particles that have been removed from the system are stored in a used-list. Furthermore, we have an unused-list that is used as a reservoir for particles that are to be injected. In this way we need not free and allocate memory for each particle that is removed or injected. Used particles are simply transferred from the inside-list to the used-list and particles that are to be injected are transferred from the unused-list to the inside-list. When the unused-list becomes empty, we rehash the particles in the used-list to give them the desired velocity distribution and place them in the unused-list. By letting the initial unused-list hold some extra particles, we can through this procedure avoid the need for any memory allocation during the simulation. As a fallback, the program can allocate more particles if the initial unused-list turned out to be too small.

The implementation with several lists is fast, and the memory overhead is not significant. However, we need to implement the lists as doubly linked lists in order to enable particles to switch lists. This implies some overhead in the memory per particle since each particle need a pointer to both the next particle and the previous particle in the list. The total memory per particle is still only 40 bytes since each particle, apart from the two pointers, only holds three keys: $z, v_{z}$, and $\mu$. 


\subsection{Particle-Fluid Coupling}

The electrons are strongly magnetized which means that they are guided by the magnetic field lines and therefore only move along $z$, although in a gyrating motion. Hence, there are $n_{x}$ independent particle pushers in the total simulation. In each particle pusher we need at least about 20 million particles to obtain reasonably good statistics when computing the temperatures from the particle distribution. For each $x$ the particles use at least about 0.8 GB of memory, and the entire simulation uses over $20 \mathrm{~GB}$, which indicates that a parallelization is needed. A huge number of particles, of course, leads to long computer times. The ratio between the time spent in a single particle pusher and the time spent in the field solver is roughly 1500 to 1 .

Since almost all time is spent in the particle pushers and there are $n_{x}$ independent pushers, this application is perfectly suited for parallelization on $n_{x}$ processors. The code, which is written in $\mathrm{C}$, is therefore parallelized using the Message Passing Interface (MPI) [4]. For each time step in the parallelized code, every processor solves (8), then each processor solves (9) and calls the particle pusher with its own value of $x$. Finally, before time is incremented, the fields in the vector $\mathbf{U}$, the temperatures and some help variables are synchronized on all processors using MPI_Allgather. This implies a message passing of about 0.2 MB for each time step.

The simulations are performed on the Sarek Linux Cluster at the High Performance Computing Center North (HPC2N) [5]. This cluster has 384 64-bit AMD Opteron 2.2 GHz CPUs in 192 dual nodes. The network, which is switched to give similar performance between each pair of nodes, has a bandwidth of about $250 \mathrm{MB} / \mathrm{s}$ and a latency of a few microseconds. With this bandwidth, the message passing will for each time step last only $10^{-3} \mathrm{~s}$, while the entire time step lasts for roughly $10 \mathrm{~s}$. Thus, the time for communication is negligible. If the load balancing between processors is good this would imply a parallelization efficiency of nearly $100 \%$, and since each processor handles roughly equal amounts of particles we have no reason to expect otherwise. The Sarek cluster also has the advantage that each processor has access to 4 GB of RAM, which implies that we can use the huge number of particles that is needed for good statistics in the temperature calculations.

\section{Discussion and Conclusions}

According to the physical results presented in [1], the particle-fluid model produces results that are consistent with observations, and the model is a major step forward compared to previous dynamic models of auroral electron acceleration, for example [6], [7], and [8], where the variation in the electron temperatures has been neglected. The implementation is, however, much more complicated compared to implementing a pure fluid model.

The model proves to be very efficient to parallelize on the same number of processors as the number of grid points in the direction perpendicular to the 
magnetic field. A further parallelization of the code can be accomplished by letting the particles at a certain field line be distributed in several linked lists and update each list on different processors. If the lengths of these lists are approximately equal, which is easily accomplished by always injecting particles into the shortest list, the load balancing of this parallelization would be perfect. Furthermore, the only communication needed in this parallelization is summing the temperatures of all lists into a single temperature along the field line, which makes this parallelization very efficient.

\section{Acknowledgments}

This research was conducted using the resources of High Performance Computing Center North (HPC2N), and was supported by the Swedish National Graduate School of Space Technology.

\section{References}

1. Vedin, J., Rönnmark, K.: Particle-fluid simulation of the auroral current circuit. J. Geophys. Res. 111 (2006), A12201, doi:10.1029/2006JA011826

2. Birdsall, C. K., Langdon, A. B.: Plasma physics via computer simulation. McGrawHill Book Company, New York (1985)

3. Degrez, G.: Implicit time-dependent methods for inviscid and viscous compressible flows, in Computational Fluid Dynamics, edited by J. F. Wendt, pp. 180-222, Springer-Verlag, New York (1992).

4. The Message Passing Interface (MPI) http://www-unix.mcs.anl.gov/mpi/

5. High Performance Computing Center North, Umeå, Sweden http://www.hpc2n.umu.se

6. Goertz, C. K., Boswell, R. W.: Magnetosphere-ionosphere coupling. J. Geophys. Res. 84 (1979) 7239-7246

7. Streltsov, A. V., Lotko, W., Johnson, J. R., Cheng, C. Z.: Small-scale, dispersive field line resonances in the hot magnetospheric plasma. J. Geophys. Res. 103 (1998), 26559-26572

8. Rönnmark, K., Hamrin, M: Auroral electron acceleration by Alfvén waves and electrostatic fields. J. Geophys. Res. 105 (2000) 25333-25344 